Fecha de recepción: diciembre 2017 Fecha de aceptación: marzo 2018 Versión final: julio 2019

\section{Prólogo. Aportes al análisis de las prácticas culturales contemporáneas de la Argentina reciente, desde la perspectiva de Pierre Bourdieu}

Laura Colabella * y Patricia Vargas **

\begin{abstract}
Resumen: Este Dossier homenajea al sociólogo francés Pierre Bourdieu, uno de los autores más citados en las ciencias sociales, a través de artículos de investigadores argentinos que retoman libros o conceptos específicos del autor-tributo para repensar sus propios temas de investigación. Específicamente propusimos a cada participante recuperar los aportes bourdeanos desde una óptica conceptual y metodológica, con especial énfasis en el trabajo de campo y el proceso de reflexividad. Para ello, convocamos a quienes, apoyados en investigaciones empíricas y/o etnográficas, den cuenta de la contribución de Bourdieu en sus procesos de producción de conocimiento social, en particular en los campos del diseño, la educación y las clases sociales.
\end{abstract}

Palabras claves: Bourdieu - Diseño - Clases Sociales - Educación.

[Resúmenes en inglés y portugués en las páginas 15-16]

${ }^{(*)}$ Laura Colabella es Dra en Antropología Social (MN-UFRJ), Profesora Asociada Regular por la UNAJ y Profesora de la Maestría en Sociología y Ciencias Políticas de FLACSO Argentina. Correo: mlauracol@yahoo.com.ar

${ }^{(*)}$ Patricia Vargas es Dra. en Antropología Social, investigadora del Centro de Antropología Social del IDES y docente titular de antropología en el programa UBA XXI, adjunta de antropología social y cultural en la UNPAZ y titular de antropología y problemas socioculturales en la Universidad de Belgrano. Dicta cursos de métodos y trabajo de campo en programas de posgrado de Argentina y Chile. Correo: patriciabeatrizvargas@gmail.com

En el presente Dossier nos proponemos homenajear al sociólogo francés Pierre Bourdieu, sin duda uno de los autores más citados en las ciencias sociales por investigadores de campos de estudios muy diversos como la educación, la cultura, la economía, el consumo, las clases sociales y la política.

Nacido en 1930 en Deguin, un pueblo del Bearn, cuenta Enrique Martín Criado en el prólogo a "Sociología de Argelia", que era nieto de aparcero e hijo de un empleado de correos. En "Autoanálisis de un sociólogo" se describe a sí mismo como un "tránsfuga" entre cam- 
pesinos, es decir, como alguien que traiciona su origen de clase a partir de su éxito escolar. Esta circunstancia es la que le permite ingresar a la Escuela Normal Superior, institución de elite intelectual donde comienza a estudiar filosofía en 1951. Ya filósofo desembarca en Argelia como soldado en 1956, pasándose con armas y bagajes a las ciencias sociales. A partir del material que recoge en esa experiencia es que empieza a trabajar en lo que será la "Sociología de Argelia". De regreso a Francia, en 1960, comienza a tomar cursos con Levi-Strauss, a aprender bereber y a hacer trabajo de campo en su Bearn natal. Estas experiencias nos permiten hablar de un Bourdieu etnógrafo por lo cual invitamos a diversos investigadores del país a presentar trabajos que discutan algunos conceptos centrales del autor, como habitus, campo, capital material y simbólico, estrategias matrimoniales, clase social y distinción y la construcción social del gusto, entre otros.

Específicamente propusimos a cada participante señalar el aporte de Bourdieu, a partir de un libro o concepto específico, para repensar sus propios temas de investigación, desde una óptica conceptual y metodológica, con especial énfasis en el trabajo de campo y el proceso de reflexividad. Para ello, convocamos a quienes, apoyados en investigaciones empíricas y/o etnográficas, den cuenta de la contribución de nuestro autor- tributo respecto de su proceso de producción de conocimiento social.

Este Dossier tuvo como antecedente las VIII Jornadas de Etnografía y Métodos Cualitativos realizadas en agosto de 2016 y organizadas por el Centro de Antropología Social del Instituto de Desarrollo Económico y Social (CAS-IDES) dirigido por Rosana Guber. En dicho evento, Laura Colabella y Patricia Vargas coordinaron el tradicional simposio que en el marco de las jornadas tributa a autores considerados clásicos de las Ciencias Sociales. En esa oportunidad, el homenaje se concentró en la figura de "El Bourdieu etnógrafo".

Una segunda instancia de discusión de los trabajos que forman parte del Dossier, tuvo lugar en julio del 2018 en el marco del Noveno Congreso Latinoamericano de Enseñanza del Diseño llevado adelante en la Universidad de Palermo.

Retomando los intercambios construidos en ambos espacios académicos, hoy celebramos la presentación de esta producción colectiva, que se inscribe en la línea de investigación "Etnografía, Cultural Material y Educación. Aportes desde las perspectivas de Pierre Bourdieu y Annette Weiner", desarrollada en la Facultad de Diseño y Comunicación de la Universidad de Palermo.

El texto que inaugura este dossier corresponde a Juan Dukuen. En la primera parte, el autor, nos presenta un recorrido arqueológico por la categoría "habitus" en los estudios sobre Sociología de la Cultura de los años 60, apuntando a mostrar que dicho término lejos de ser un aparato teórico sin historia producido en bloque por generación espontánea-como solían señalar sus detractores calificándolo como un autor "determinista"- es más bien una herramienta teórico-metodológica para criticar, reelaborar e investigar empíricamente. Su contribución concluye con la aplicación del término a una investigación empírica acerca de los vínculos de los jóvenes con la política en colegios para "clases altas y elites" de Buenos Aires y área metropolitana. Su trabajo de campo se localiza en una escuela privada bilingüe de tradición británica situada en un barrio de clases altas de la Ciudad de Buenos Aires. De esa manera, Dukuen devuelve a la categoría su valor de uso no dogmático.

El segundo, artículo pertenece a Victoria Gessaghi y Alicia Méndez, quienes a partir del libro "La Nobleza de Estado" de Pierre Bourdieu, analizan cómo opera el "efecto dignidad" 
otorgado por títulos en dos colegios secundarios. El primero de ellos de gestión privada y destinado a la "clase alta". El segundo, estatal y universitario: el Colegio Nacional de Buenos Aires; donde un sistema estricto de ingreso en base a un reclutamiento heterogéneo daba un cierto margen para que accedan individuos de zonas desfavorecidas. El trabajo resulta en una contribución novedosa para releer un texto que pertenece a la producción de la fase final de Bourdieu, desde la mirada de otro sociólogo francés, Bernard Lahire, y desde los aportes de la evidencia empírica de cada una de las investigadoras.

El tercer artículo es autoría de las coordinadoras, Laura Colabella y Patricia Vargas. Quienes desde la lectura del sociólogo francés Loïc Wacquant de la experiencia de proximidad y distancia en sendos trabajos de campo de Pierre Bourdieu en Argelia y su Bearn natal, muestran cómo estudiantes de las Universidades Nacionales Arturo Jauretche y José C. Paz, localizadas en los Municipios de Florencio Varela y José C. Paz, construyen la alteridad al ser compelidos a hacer trabajo de campo en espacios distantes de sus hogares. El artículo se detiene en las condiciones y los modos en que los estudiantes hacen trabajo de campo y redactan sus etnografías. Revelando que "hacer trabajo de campo", más que delimitar un área geográfica, supone para ellos, desplegar una red de relaciones que articula la universidad con sus barrios y con espacios y grupos localizados en la Ciudad de Buenos Aires y el interior del país.

El cuarto artículo, corresponde a la contribución de Lorena Schiava D'Albano, quien recupera el libro "El baile de los solteros". En ese trabajo, los conceptos de "habitus" y "Hexis corporal" cobran protagonismo, al ser mostrados en el modo en que algunos campesinos del Bearn que van al baile, quedan fuera de la pista, aislados, observando al resto bailar. Su explicación pone el foco en el cuerpo, peso, talla, volumen y al modo en que interiorizaban técnicas corporales que daban lugar a que algunos fueran percibidos con "aspecto campesino" y perdieran atractivo para las mujeres de la región. Esta explicación inspira a la autora para mostrar cómo en un curso de Asesoría de Imagen, en el barrio de Recoleta, al que ella misma asistió en calidad de alumna para iniciar su investigación empírica, la noción de cuerpo va cediendo paso a la noción de persona.

En quinto lugar, Alicia Gutiérrez y Héctor O. Mansilla con base en la teoría de la práctica bourdesiana, en tanto superación del sentido objetivo y el sentido vivido, muestran aspectos de la "cocina de la investigación" de un estudio que llevan adelante desde hace varios años, acerca de la reproducción social en un barrio de la ciudad de Córdoba. Su minucioso análisis de los sentidos vividos por los sujetos en el barrio analizado es un puente más que interesante para llegar a los esquemas de acción y percepción que éstos ponen en juego en sus prácticas cotidianas.

En sexto lugar, Florencia Blanco Esmoris a partir del análisis de la lógica binaria implícita en el análisis espacial de la casa Kabila de Pierre Bourdieu, se propone mostrar el uso del espacio por parte de una familia de "clase media", que habita en una casa reciclada en varias etapas y ubicada en una localidad de la zona oeste del Gran Buenos Aires. El texto, que se detiene en descripciones minuciosas acerca de la apropiación estacional y diaria de ciertos espacios de la casa - "arriba y abajo" y "adentro y afuera"- por parte de los miembros de la "familia de Gloria"; da cuenta también de las lógicas implícitas en esa apropiación. Las cuales deben ser frecuentemente renovadas porque la casa, al decir de la autora, está en constante devenir o, según sus habitantes "no lo van a terminar más". 
Posteriormente, el lector se encontrará con cuatro textos que, inspirados en el Bourdieu que analiza la producción cultural, nos proponen mirar de cerca la experiencia del diseño en Buenos Aires durante los últimos 20 años.

En esta línea de trabajo, Paula Miguel presenta un recorrido en torno al proceso colectivo de producción (y posicionamiento público) del diseño de indumentaria (y su valor) en la escena local, con énfasis en sus inicios en el año 2000. A partir del análisis de diversos procesos confluyentes - profesionalización e institucionalidad académica; acción colectiva de diseñadores que se consolidan en el campo en términos de productores de "diseño de autor"; visibilización pública de los productos de diseño, de los circuitos de comercialización y de los eventos específicos de exposición (tales como el BAF Week), y el papel destacado que cumple la prensa escrita en dicho proceso-, Miguel se propone mostrar -retomando conceptos centrales de Bourdieu-cómo juega el capital simbólico, al interior del diseño como campo. Su perspectiva multidimensional apunta a comprender cómo "hacerse un nombre" en el mundo del diseño se convierte en "capital de consagración", y, aunque aparentemente pareciera un trabajo individual ("diseño de autor") depende del conjunto de prácticas que se dan entre productores, consumidores y múltiples mediadores entre ambos.

Por su parte, María Eugenia Correa muestra un derrotero análogo, pero respecto del diseño industrial. Centrándose en las trayectorias de miembros de las primeras camadas de diseñadores industriales egresados de la Universidad de Buenos Aires (a partir de los años noventa), deja al descubierto cómo la "autogestión" y la inserción laboral, es un claro indicador de la debilidad estructural en la oferta de puestos de trabajo vinculados con la incumbencia profesional por excelencia de la disciplina -el diseño de productos para el sector industrial-. Esta capacidad de producir acciones no previstas por los ideales tradicionales del diseño industrial y otorgarle legitimidad al interior del campo, son interpretadas por la autora, a partir de la dimensión inventiva del habitus que les permite a los diseñadores industriales co-producir una illusio novedosa por la que vale la pena jugar dicho juego social.

Posteriormente dos autoras retratan el devenir del mundo del diseño en la última década: Bárbara Guerschman, analizando los modos de producción y comercialización que se organizan a través de las colecciones estacionales entre los diseñadores de la Ciudad de Buenos Aires (Palermo) y Gabriela Alatsis, analizando los procesos de consolidación reciente de los diseñadores en la zona sur del Gran Buenos Aires (Quilmes). Guerschman, a partir de las reflexiones bourdeanas en torno a los campesinos argelinos y los cambios en la concepción temporal a que se ven compelidos por su ingreso abrupto al capitalismo, producto del proceso colonizador y sus consecuencias, inspira sus reflexiones en torno a la profesionalización de los diseñadores cuando se transforman en una "marca de diseño". Alatsis, por su parte, se concentra en el papel central que jugó el programa Diseños Al Sur (DAS) como política pública implementada para jerarquizar y consolidar el diseño en el Municipio de Quilmes (vigente entre el 2008 y el 2015). Valiéndose de los análisis de Bourdieu respecto del papel de los intermediarios culturales, la autora muestra detalladamente el proceso por el cual las coordinadoras del DAS coadyuvaron a la instalación y posicionamiento de un polo de diseño en un espacio social que no contaba con experiencia previa al respecto. Justamente los esfuerzos del programa se orientaron a impulsar la comerciali- 
zación de productos de diseño a la vez que producir un sistema de creencias que otorgase valor (simbólico y económico) y legitimidad social a dichas producciones.

Por último, dos trabajos recuperan a la par que discuten las ideas bourdeanas vertidas en “La Distinción”. María Eugenia Correa y Matías Romani analizan los significados sociales que adquiere consumir tecnología de Apple en Argentina durante el período 2015-2018, en el marco de un contexto de circulación restringida de dichos bienes. La tensión entre el carácter restringido de su circulación en nuestro país a la vez que la producción masiva y global de este tipo de producto cultural ponen en jaque la idea lineal del consumo distintivo asociado a la clase social, agregándole matices y especificidad al análisis que nos proponen estos autores. Cierran el Dossier, Victoria Irisarri y Nicolás Viotti, quienes proponen una relectura de Bourdieu a partir del análisis de prácticas específicas (estético-musicales tales como la cumbia electrónica y religioso-espirituales tales como la meditación) ilustrativas de lo que ellos denominan un modo de vida vanguardista, cosmopolita e innovador de un sector de las clases medias urbanas de Buenos Aires. Contrario a interpretar que la participación en estos circuitos podría significar exclusivamente marcas de distinción, los autores muestran exhaustivamente cómo expresan formas de adhesión relacionadas con las biografías y vinculación "transversal y fragmentada", que debe ser comprendida (y aprehendida) contextualmente.

\title{
Bibliografía:
}

Criado, M. (2006). “Introducción” en Bourdieu, P. Sociología de Argelia y tres estudios de etonología Cabilia, Madrid, CIS.

Bourdieu, P. (2004). Autoanálisis de un sociólogo. Madrid, Anagrama.

\begin{abstract}
This Dossier pays homage to the French sociologist Pierre Bourdieu, one of the most cited authors in the social sciences, through articles by Argentine researchers who take up books or specific concepts of the author-tribute to rethink their own research topics. Specifically, we proposed each participant to recover the contributions from a conceptual and methodological perspective, with special emphasis on field work and the process of reflexivity. To do this, we call on those who, supported by empirical and / or ethnographic research, to give an account of Bourdieu's contribution in his processes of social knowledge production, particularly in the fields of design, education and social classes.
\end{abstract}

Keywords: Bourdieu - Design - Social Classes - Education.

Resumo: Este Dossiê é uma homenagem ao sociólogo francês Pierre Bourdieu, um dos autores mais citados nas ciências sociais, através de artigos de pesquisadores argentinos que adotam livros ou conceitos específicos do autor-tributo para repensar seus próprios tópicos de pesquisa. Especificamente, propusemos a cada participante recuperar as con- 
tribuições de uma perspectiva conceitual e metodológica, com ênfase especial no trabalho de campo e no processo de reflexividade. Para tanto, convocamos aqueles que, apoiados em pesquisas empíricas e / ou etnográficas, dão conta da contribuição de Bourdieu em seus processos de produção de conhecimento social, particularmente nas áreas de design, educação e classes sociais.

Palavras chave: Bourdieu - Design - Classes Sociais - Educação.

[Las traducciones de los abstracts fueron supervisadas por el autor de cada artículo] 